\title{
Probe current, probe size, and the practical brightness for probe forming systems
}

\author{
M. S. Bronsgeest ${ }^{\text {a) }}$ and J. E. Barth ${ }^{\text {b) }}$ \\ Delft University of Technology, Lorentzweg 1, 2628 CJ Delft, Netherlands \\ L. W. Swanson \\ FEI Company, Hillsboro, Oregon 97124 \\ P. Kruit \\ Delft University of Technology, Lorentzweg 1, 2628 CJ Delft, Netherlands
}

(Received 20 December 2007; accepted 18 March 2008; published 29 April 2008)

\begin{abstract}
Probe size, shape, and current are important parameters for the performance of all probe forming systems such as the scanning (transmission) electron microscope, the focused ion beam microscope, and the Gaussian electron beam lithography system. Currently, however, the relation between probe current and probe size is ill defined. The key lies in a lacking definition of "size." This problem is solved with the introduction of the "practical brightness." In literature, many different definitions of "brightness" can be found, but for systems in which the whole of the virtual source is imaged onto the target, it is the practical brightness of a source that determines how much current is in the probe. This means that only with the practical brightness the performance of a probe forming system can be calculated quantitatively. The beauty of the practical brightness is that this source property is unaffected by the quality of the column: without interactions between electrons in the beam, the practical brightness is conserved down to the target. This makes it the only relevant brightness for probe forming systems to be used to compare different sources. The practical brightness can be measured, but can also be calculated when the source intensity profile is known. The Gaussian source intensity profile of thermionic, Schottky, and cold field emitters yields a practical brightness of $1.44 e j / \pi\langle\varepsilon\rangle$, where $j$ is the current density on the emitting surface and $\langle\varepsilon\rangle$ is the average tangential electron energy. () 2008 American Vacuum Society. [DOI: 10.1116/1.2907780]
\end{abstract}

\section{INTRODUCTION}

Probe size, shape, and current are important parameters for the performance of all probe forming systems such as the scanning (transmission) electron microscope, the focused ion beam microscope, and the Gaussian electron beam lithography system. Probe size and current are interrelated. For example, for high resolution work, a small probe is desirable, but the probe cannot be too small because the number of electrons or ions in the probe must be sufficient to actually see something. Currently, however, it is not possible to accurately calculate the current in a probe, because the probe current-probe size relation is ill defined.

We recall that the probe at the target is a demagnified image of the source that is blurred and enlarged to some extent by diffraction and lens aberrations. Note that, in this respect, "source" is not the physical emitting surface, but the real or virtual crossover formed by the field near the emitting tip. ${ }^{1}$ Important to the amount of current in the probe at the target is how many particles the source can emit per unit area per solid angle per second (particle or charge flux density per unit solid angle). In electron and ion optics, this quantity is the "richtstrahlwert" or "brightness" in $\mathrm{A} / \mathrm{m}^{2} \mathrm{sr}^{2}$ "The analogy in radiometry is brightness or "radiance" in $\mathrm{W} / \mathrm{m}^{2} \mathrm{sr}$.

\footnotetext{
${ }^{a)}$ Electronic mail: m.s.bronsgeest@tudelft.nl

${ }^{b)}$ Deceased Feb. 18, 2008.
}

Because beam brightness changes when the beam enters a section of different potential (in radiometry, a medium of different index of refraction $n$ ), the beam brightness at the source does not say much about the amount of current in the probe at the target, nor does the beam brightness at the target say anything about the quality of the source. Calling the beam brightness at the target the "source brightness" 3 or "gun brightness" ing.

The relevant source property for probe forming systems is the "richtstrahlwert-per-volt", or "normalized" or "reduced brightness" " which is the brightness divided by the beam potential in $\mathrm{A} / \mathrm{m}^{2} \mathrm{sr} \mathrm{V}$. The reduced brightness for infinitely small area and solid angle is the differential reduced brightness. This source property is conserved throughout the column (Liouville's theorem, analogy in radiometry: conservation of brightness divided by $n^{2}$ ) as long as there are no interactions between the particles in the beam, and is the bridge between source performance and the amount of current in the probe.

In general, the differential reduced brightness $B_{\text {diff }}$ is a function of the position in the plane, $\vec{r}$, and of the beam direction with respect to the normal of the plane, $\vec{t}$, (the generalized brightness function in Hawkes and Kasper ${ }^{2}$ ). The current in the specimen plane, $I_{p}$, can thus be calculated from 


$$
I_{p}=V_{p} \int_{\Omega} \int_{A} B_{\mathrm{diff}}(\vec{r}, \vec{t}) d \vec{r} d \vec{t},
$$

where $V_{p}$ is the beam energy at the specimen and the integration is to be performed across the total area of the beam intensity distribution in the specimen plane and for all angles that arrive at the specimen. The beam current that hits the specimen is determined by a current-limiting aperture. Usually, this aperture cuts out a very small portion of the total emission cone which allows for the approximation of uniform current density in the aperture plane and uniform angular intensity in the (virtual) source plane and the source image plane. For the source image plane, this simplifies Eq. (1) to

$$
I_{p}=\Omega_{p} V_{p} \int_{\text {image }} B_{\text {diff }}(\vec{r}) d \vec{r},
$$

which gives the current in the source image plane. Note that the integration is performed across the total area of the (virtual) source image, not the total area of the probe, since the probe also has contributions from diffraction and aberrations.

The equation (2) expression for the probe current does not yet contain the source image area $A_{\mathrm{I}}$. For practical purposes, we would like to have a relation such as

$$
I_{p}=\Omega_{p} V_{p} A_{\mathrm{I}} B_{\text {pract }} .
$$

This requires a definition of $B_{\text {pract }}$ as

$$
B_{\text {pract }}=\frac{\int_{A} B_{\text {diff }}(\vec{r}) d \vec{r}}{A_{\text {pract }}} .
$$

For the source image plane, the integration in Eq. (4) would be over the total source image area, and $A_{\text {pract }}$ would be a practical measure for the size of the source image: $A_{\mathrm{I}}$ $=\pi\left(d_{\mathrm{I}} / 2\right)^{2}$ with $d_{\mathrm{I}}$ a practical source image diameter measure. There are many choices for $d_{\mathrm{I}}$ : full width half maximum (FWHM), full width containing $50 \%$ of the current (FW50), root mean square (rms), etc. By choosing a measure for $d_{\mathrm{I}}$ Eq. (4) becomes a practical brightness, which can be used to finally quantify the relation between probe current and source image size.

The source image is the (de)magnified image of the (virtual) source with diameter $d_{v}$ : thus $d_{\mathrm{I}}=M d_{v}$ with $M$ the column magnification. The practical brightness is the same in both planes. By applying the definition of Eq. (4) to the plane of the virtual source, the practical brightness can be written as

$$
B_{\text {pract }}=\left[\frac{\int_{A} B_{\mathrm{diff}}(\vec{r}) d \vec{r}}{A_{\text {pract }}}\right]_{\text {source }}=\frac{4 I^{\prime}}{\pi d_{v}^{2} V_{\text {ext }}},
$$

with $I^{\prime}$ the angular intensity of the (virtual) source, $I / \Omega$, measured at the extractor, $V_{\text {ext }}$ the extraction voltage, and $d_{v}$ the diameter of the (virtual) source. Crewe ${ }^{7}$ has also recognized Eq. (5) as the only meaningful brightness with respect to probe forming systems, although he does not give a definition of $d_{v}$. In Sec. II, the definition of the practical brightness will be completed with our choice of $d_{v}$. The practical brightness of a source can be (partly) determined by Coulomb interactions taking place between emitter and extractor. This is addressed in Sec. III. Section IV discusses briefly the conservation of the practical brightness of the source in a column. When in a system, the practical brightness of the source is conserved down to the target, the relation between the probe current and source image size [Eq. (3)] can be quantified. This, of course, requires the practical brightness of the source. How one could measure the practical brightness of a source is touched in Sec. V. Section VI gives the derivation of the practical brightness for the particular case of thermionic, Schottky, and cold field emission electron sources in terms of their basic emission properties. Because a real probe contains not only the source image size but also contributions from aberrations and diffraction (electron sources), it is shown in Sec. VII how the different contributions can be added in order to get the relation between probe current and total probe size.

\section{DEFINING SIZE}

To complete the practical brightness definition in Eq. (5), we have to choose a measure for diameter $d_{v}$. For that, we look at measures for the total probe size $d_{p}$, because it makes sense to use the same size measure for the virtual source as for the total probe that contains the image of that virtual source. Reimer ${ }^{8}$ has pointed out that a definition of electronprobe size should be free of assumptions about the electronprobe profile. This excludes the often used FWHM. Jansen ${ }^{9}$ explains why the rms is unsuitable. A measure that assumes no particular shape of the probe intensity profile is the diameter $d(\mathrm{FC})$ containing a fraction of the current (FC). Usually, this is sufficient information: not the exact profile of the probe, but the number of electrons within a certain area is what matters.

For the value of $\mathrm{FC}$, we choose $\mathrm{FC}=0.5$, which is a convenient choice for a number of reasons. One is the relation between probe size and a common measure for resolution in scanning electron microscopy (SEM): the knife edge. Edge detection is often used to indicate resolution. In that method, the probe is swept over an edge and the current behind the edge or on the edge is detected while it rises from $0 \%$ to $100 \%$. A common measure for resolution is the distance between the $25 \%$ and $75 \%$ levels of the current. The relation between the measured knife edge width and the diameter of the probe will, however, depend on the shape of the probe intensity distribution and the definition of probe diameter. Figure 1 shows for three different intensity distributions the ratio of the probe diameter and the knife edge width, $d(\mathrm{FC}) / d_{25-75}$, for different definitions of the diameter as defined by FC. As anticipated this ratio is a function of FC, and also depends on the particular probe shape. There is, however, an intersection of the three curves very close to FC $=0.5$. This means that (only) for $\mathrm{FC}=0.5$, one can conveniently convert between edge resolution and probe size without having to know the shape of the probe: $d_{\text {FW50 }}$ $=1.76 d_{25-75}$. The inset in Fig. 1(b) shows the $d_{\text {FW50 }}$ and $d_{25-75}$ for a top hat intensity distribution. 

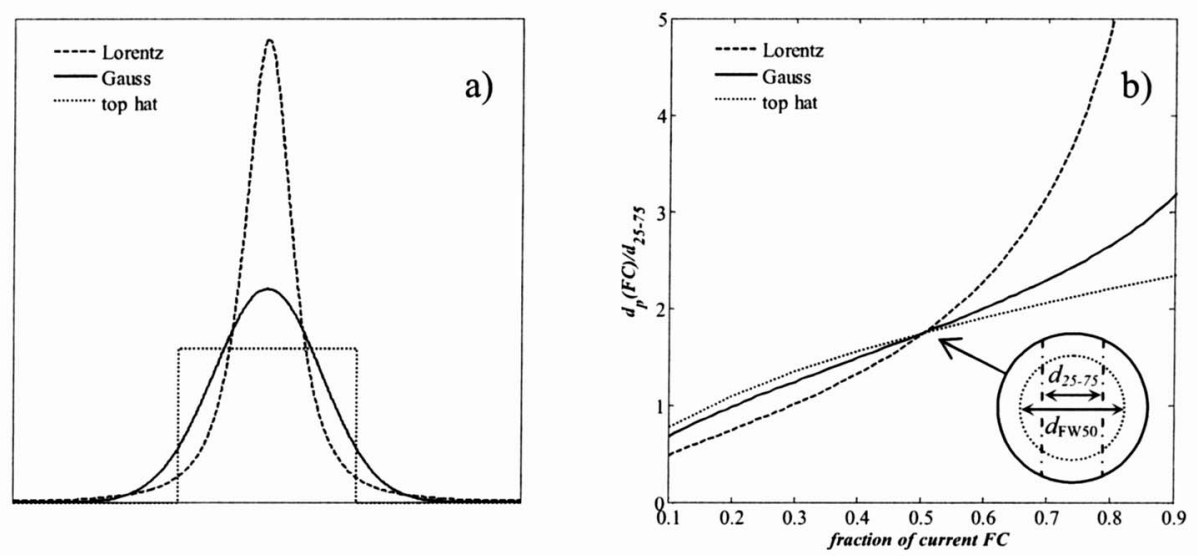

FIG. 1. (a) Cross section through the probe normalized for content for Gaussian, Lorentzian, and top hat distribution (2D). (b) Ratio of the probe size $d_{p}(\mathrm{FC})$ and the $25 \%-75 \%$ edge resolution, $d_{25-75}$, for different current fractions. The inset shows the $d_{\text {FW50 }}$ and $d_{25-75}$ for a top hat intensity distribution.

Two additional arguments follow from the point that it makes sense to use the same size measure not only for the virtual source and the total probe but also for all contributions to the total probe. In that case, it is convenient that the FW50 of the chromatic aberration contribution, contrary to, e.g., the FWHM, is approximately independent of the shape of the energy distribution. ${ }^{10}$ Also, for electron probes, the inevitable diffraction contribution [the intensity distribution produced by Fraunhofer diffraction around a circular aperture (Airy pattern)], has a FW50 width that is very close to its FWHM (4\% difference).

Finally, the probe intensity profiles encountered in practice are often approximately Gaussian, for which the FW50 equals the FWHM. This should facilitate adoption of the FW50. Thus, with $d_{v}$ in Eq. (5) taken to be the FW50 value, the practical brightness definition is complete and we can drop the subscript "pract."

\section{COULOMB INTERACTIONS CLOSE TO THE EMITTER}

When the particle density in a beam is high, Coulomb interactions between the individual particles disturb the longitudinal and transverse velocity distributions. ${ }^{9}$

This means that when a source emits with a high particle density, the properties of the beam will start changing right after emission. This is the case, e.g., for liquid metal ion sources. ${ }^{11}$ Due to the interactions, the virtual source size will grow traveling from emitting surface to extractor. The increased virtual source size as seen by looking back from the extractor plane is the source size used in the practical brightness of Eq. (5). For these sources, their practical brightness is determined by the Coulomb interactions occurring between the emission site and the extractor.

For thermionic, Schottky, and cold field emitters, the effect of Coulomb interactions up to the extractor is often negligible. In that case, the virtual source size is determined by emission properties of the surface and the geometry of the emitter-extractor module. In Sec. VI, it will be shown that the practical brightness is then directly proportional to the current density at the emitting surface, which can be calculated from electron emission theory. Unfortunately, it is so that the higher this "intrinsic" practical brightness, the more important the effect of Coulomb interactions becomes. For Schottky emitters, it has been shown that interactions become important when the intrinsic practical brightness exceeds $\sim 3 \times 10^{8} \mathrm{~A} / \mathrm{m}^{2}$ sr V. ${ }^{12}$ For the new source types based on carbon nanotubes ${ }^{13}$ and nanotips, ${ }^{14}$ we expect their practical brightness to be largely determined by the Coulomb interactions.

\section{CONSERVATION OF PRACTICAL BRIGHTNESS IN THE COLUMN: RELATION PROBE CURRENT-SOURCE IMAGE SIZE}

By careful design of the column, the effect of (additional) Coulomb interactions in the column can be minimized. ${ }^{15}$ If the effect is negligible, the practical brightness is conserved from the extractor down to the target, unless the source image intensity profile is changed. The intensity profile is changed, e.g., when the source is imaged onto a beamlimiting aperture. The aperture cuts off the tails of the current density distribution in the image thereby changing the source profile. As a result, part of the current is lost but the new FW50 diameter will be smaller than the original FW50 diameter and the practical brightness will increase. This can be of practical importance in mitigating the brightness loss that occurs in a monochromator.

If the practical brightness at the extractor is conserved down to the target, we can quantify the relation between probe current and source image size [Eq. (3)]:

$$
I_{p}=\pi \alpha_{p}^{2} V_{p} \frac{\pi}{4} d_{\mathrm{I}}^{2} B
$$

with $\alpha_{p}$ the half-opening angle of the beam at the target and $B$ determined by the source properties [Eq. (5)].

\section{HOW TO GET THE PRACTICAL BRIGHTNESS OF A SOURCE}

The practical brightness of a source can be evaluated when its angular intensity and FW50 virtual source diameter are known at a particular extraction voltage [Eq. (5)]. 
The FW50 virtual source diameter can be calculated from the shape and size of the intensity profile of the virtual source. For some source types, this is known and does not need to be measured: thermionic emitters, e.g., have a Gaussian source intensity profile with a temperature dependent width. ${ }^{16}$ For other source types, only their shape is known, such as for liquid metal ion sources, which have a shape with much stronger tails than the Gaussian, ${ }^{17}$ or both shape and size are unknown, such as for the nanotube ${ }^{13}$ or the nanotip ${ }^{14}$ emitter. In that case, the practical brightness of the source can only be found by measuring the source intensity profile.

There is an ongoing effort to measure the size and shape of probes in general. The most commonly used technique for spot size measurement in probe forming systems is the knife edge scan. This method generally works for probe sizes down to a few nanometer, ${ }^{18,19}$ although it has potential sources of error. ${ }^{20} \mathrm{~A}$ different method is using a point projection microscope, ${ }^{13,21}$ but to extract a size from the experimental Fresnel fringe patterns, one has to assume the shape of the source intensity distribution. Also, Fourier methods have been applied, ${ }^{22}$ but a routine that can deal with unknown probe shapes is still under development. ${ }^{20,23,24}$ Perhaps, the most straightforward method to obtain the size and shape of a virtual electron source is to put the source as emitter in a transmission electron microscope and use the column to make a highly magnified image of the source. ${ }^{25}$

Once the probe size is known, it still includes contributions from diffraction and aberrations in addition to the desired source image intensity profile. However, when the latter dominates the probe, the total size can be corrected with the root power sum (RPS) method (Sec. VII) to yield the size of the virtual source.

\section{PRACTICAL BRIGHTNESS FOR THERMIONIC, SCHOTTKY, AND COLD FIELD EMISSION ELECTRON SOURCES}

The practical brightness of thermionic, Schottky, and cold field electron emitters is a special case, because it can be shown to be directly proportional to the current density at the emitting surface, which follows from electron emission theory when local work function, temperature, and field are known. This is only when the effect of Coulomb interactions between these types of emitters and the extractor is negligible, which often is a safe assumption.

The starting point is Eq. (5). To derive expressions for the angular intensity $I^{\prime}$ and virtual source size $d_{v}$, we first need the position of the virtual source. The position of the virtual source can be found by tracing back from the extractor the tangents to the trajectories of electrons that have left the emitter normal to the surface from an off-axis position with zero tangential energy ("cold" electrons). The virtual source plane is located where these tangent lines cross the optical axis (Fig. 2).

A relation between the angular current density at the extractor, $I^{\prime}$, and the current density on the emitting surface, $j$, can be calculated from electron emission theory for infinite planes if the field, work function, and the temperature at the
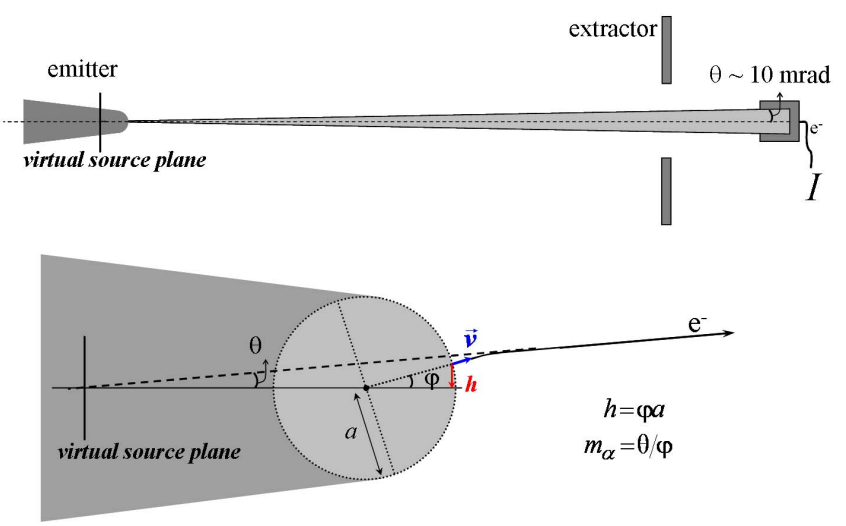

FIG. 2. Schematic representation of tip-extractor geometry and close up of tip end to illustrate the position of the virtual source. The trajectory is for a cold electron: one emitted perpendicular to surface with zero tangential energy. Paraxial approximation is used.

surface are known. ${ }^{1}$ In the paraxial approximation, the relation between $I^{\prime}$ and $j$ is found from the trajectories of cold electrons:

$$
I^{\prime}=\frac{I}{\pi \theta^{2}}=\frac{I}{\pi m_{\alpha}^{2} \varphi^{2}}=\frac{I a^{2}}{\pi h^{2} m_{\alpha}^{2}}=j\left(\frac{a}{m_{\alpha}}\right)^{2},
$$

where $a$ is the tip radius, $h$ is the distance between the optical axis and the launch position, $\theta$ is the angle of the trajectory at the extractor, and $\varphi$ is the "internal" angle (Fig. 2). The ratio of the angles is the so-called angular magnification $m_{\alpha}{ }^{1,26}$ For spherical tip ends, Tuggle et al. have found that $m_{\alpha}$ is constant for internal angles up to at least $10^{\circ}$.

Whereas the position of the virtual source was found by tracing back electrons with zero tangential energy, the size of the virtual source is determined by electrons emitted with nonzero tangential energies. This is illustrated in Fig. 3. These electrons do not cross the optical axis in the plane of the virtual source.

In the paraxial approximation, using the conservation of phase space, the distance from the optical axis in the virtual source plane, $\rho$, of an electron emitted with tangential energy $\varepsilon$, can be found from ${ }^{27-29}$

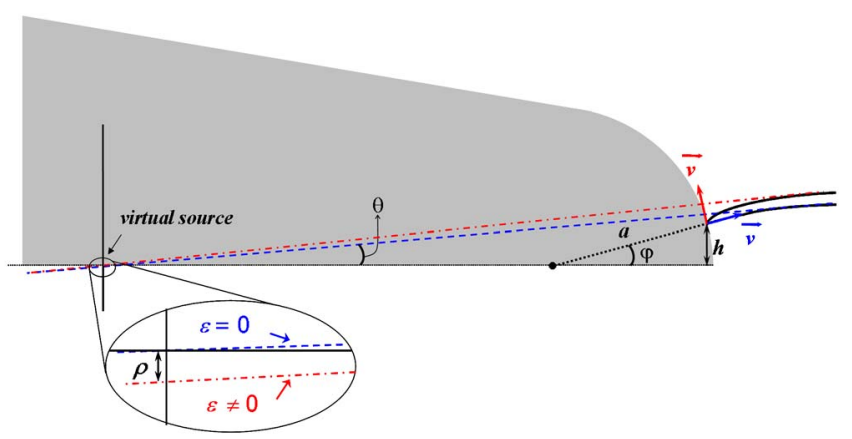

FIG. 3. An electron with nonzero tangential energy does not cross the optical axis in the plane of the virtual source, as does a cold electron with zero tangential energy. 


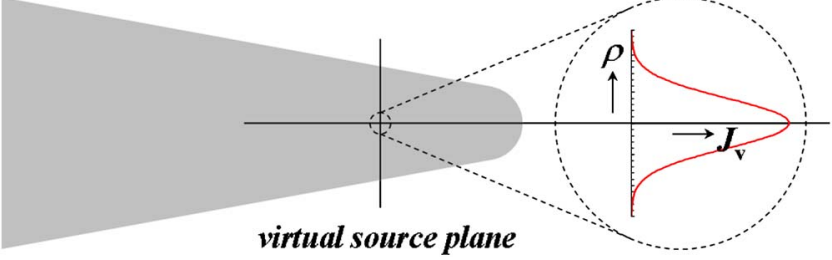

FIG. 4. Gaussian intensity distribution in the virtual source plane.

$$
\begin{aligned}
\underset{\text { emitting surface }}{\left[\Delta x \Delta p_{x}\right]} & =\underset{\text { virtual source }}{\left[\Delta x \Delta p_{x}\right]} \Leftrightarrow \underset{\text { emitting surface }}{\left[h m \nu_{t}\right]} \\
& =\underset{\text { virtual source }}{\left[\rho m\left(\theta v_{\text {ext }}\right)\right]} \Leftrightarrow h \sqrt{\varepsilon}=\rho \theta \sqrt{e V_{\text {ext }}},
\end{aligned}
$$

with $m$ is the electron mass, $e$ is the electron charge, $v_{t}$ is the tangential velocity, and $v_{\text {ext }}$ and $V_{\text {ext }}$ are the velocity and potential at the extractor. Using Fig. 2, this can be written as

$$
\rho=\frac{a}{m_{\alpha}} \sqrt{\frac{\varepsilon}{e V_{\mathrm{ext}}} .}
$$

Fujita and Shimoyama ${ }^{30-32}$ have pointed out that the paraxial lens theory is not directly applicable to electron gun analysis, because electron trajectories can have large initial angles with the optical axis. In their more generally applicable canonical mapping theory (CMT), the $a / m_{\alpha}$ factor in Eqs. (7) and (9) is replaced with the electron gun focal length $f$.

The distribution of $\rho$ in the virtual source plane is thus a projection of the tangential velocity distribution of the emitted electrons. Inside the metal, the total energy distribution is the Fermi-Dirac distribution. Outside the metal, it is different: only electrons with enough (normal) energy to surmount or tunnel through the potential barrier at the surface can escape. The tangential energy is conserved upon emission.

For thermionic and Schottky emissions, the electrons emitted are electrons from the high energy tail of the FermiDirac distribution in the metal, for which the MaxwellBoltzmann (MB) distribution is a good approximation. For a Maxwell-Boltzmann distribution, the normal and tangential velocities are independent and normally distributed (Gaussian) with a standard deviation of $\sqrt{ }(k T / m)$. The tangential energy distribution is an exponential function with a mean tangential energy of $k T$, with $T$ the temperature, and $k$ the Boltzmann constant.

For cold field emission, the electrons emitted are tunneling electrons with energies close to the Fermi energy. The $\mathrm{MB}$ approximation is no longer valid, nor is the assumption that the tangential distribution is independent of the normal distribution. It can be shown, however, that, in practical systems, the exponential function is a reasonable approximation of the tangential energy distribution also for cold field emitters, with a mean tangential energy $d=e \hbar F / \sqrt{ }(8 m \phi)$, with $F$ the electric field, $\phi$ the work function, and $\hbar$ the reduced Planck constant. $^{28,33}$

The projection of the Gaussian tangential velocity distribution at the emitting surface onto the virtual source plane gives a Gaussian spatial current density distribution $J_{v}$, (Fig. 4):

$$
J_{v} \propto e^{-\left(\rho / \rho_{T}\right)^{2}}
$$

where $\rho_{T}$ is the distance from the optical axis in the virtual source plane of an electron with the mean tangential energy $\langle\varepsilon\rangle(\langle\varepsilon\rangle$ is $k T$ for thermionic and Schottky emitters and $d$ for cold field emitters).

To evaluate the practical brightness, we need the diameter $d_{v}$ of the disk that contains $50 \%$ of the total current in $J_{v}$ :

$$
d_{v}=2 \rho_{\mathrm{FC}=0.5}=2 \frac{a}{m_{\alpha}} \sqrt{\frac{\varepsilon_{\mathrm{FC}=0.5}}{e V_{\mathrm{ext}}}} .
$$

$\varepsilon_{\mathrm{FC}=0.5}$ can be expressed in terms of the known mean tangential energy $\langle\varepsilon\rangle$ from

$$
\begin{aligned}
0.5= & \frac{\int_{0}^{2 \pi} \int_{0}^{\rho_{\mathrm{FC}=0.5}} e^{-\left(\rho / \rho_{T}\right)^{2}} \rho d \rho d \theta}{\int_{0}^{2 \pi} \int_{0}^{\infty} e^{-\left(\rho / \rho_{T}\right)^{2}} \rho d \rho d \theta}=\frac{\int_{0}^{\varepsilon_{\mathrm{FC}=0.5}} e^{-\varepsilon /\langle\varepsilon\rangle} d \varepsilon}{\int_{0}^{\infty} e^{-\varepsilon /\langle\varepsilon\rangle} d \varepsilon}=1 \\
& -e^{-\varepsilon_{\mathrm{FC}=0.5} /\langle\varepsilon\rangle},
\end{aligned}
$$

for which we have used the proportionalities $\rho^{2} \propto \varepsilon \& \rho_{T}^{2}$ $\propto\langle\varepsilon\rangle$. This gives

$$
\varepsilon_{\mathrm{FC}=0.5}=\langle\varepsilon\rangle \ln 2 .
$$

Combining Eqs. (11) and (13), the virtual source size $d_{v}$ containing $50 \%$ of the current is

$$
d_{v}=1.67 \frac{a}{m_{\alpha}} \sqrt{\frac{\langle\varepsilon\rangle}{e V_{\mathrm{ext}}}} .
$$

Using Eqs. (7) and (14) in Eq. (5) gives for the practical brightness of thermionic and Schottky sources:

$$
B=1.44 \frac{e j}{\pi k T}
$$

and for the practical brightness of cold field emitters:

$$
B=1.44 \frac{e j}{\pi d}
$$

Note that the tip radius and angular magnification are no longer required for the practical brightness: the practical brightness of these sources follows from the electron emission theory for infinite planes.

However, if the relation between current density on the emitting surface and the angular intensity at the extractor is known [Eq. (7)], only the angular intensity is required to verify the practical brightness experimentally:

$$
B=1.44 \frac{e I^{\prime}}{\pi k T}\left(\frac{m_{\alpha}}{a}\right)^{2} .
$$

The factor $m_{\alpha} / a$ (or its equivalence in CMT: ${ }^{30-32} 1 / f$ ), can be found from SEM imaging and ray tracing simulations.

The factor 1.44 in the brightness equations is a surprising, new result. Note that the quantities $e j /(\pi k T)$ and $e j /(\pi d)$ in Eqs. (15) and (16) are the often quoted axial differential reduced brightness values $B_{\text {diff }}(0)$ of thermionic and Schottky sources, and cold field emitters, respectively. ${ }^{2}$ The differential axial reduced brightness $B_{\text {diff }}(0)$ is the current from an infinitesimal area at the center of the source intensity 
profile into an infinitesimal solid angle. Remember that when electron sources are used for probe formation, the differential axial reduced brightness is not the relevant parameter because in probe formation, the whole of the virtual source is imaged. The practical brightness is proportional to the differential axial reduced brightness, but the proportionality factor depends on the shape of the source intensity profile. $B_{\text {diff }}(0)$ can therefore not replace $B$ to calculate the probe current with Eq. (6) as is done in Ref. 31 nor should FW50 brightness measurements be compared to the differential values as is done in Ref. 25. As discussed before, the practical brightness can be increased (at the cost of beam current) by imaging the source onto a beam-limiting aperture that cuts off the tails of the intensity distribution. This method can increase the practical brightness up to a maximum of $2 e j /(\pi k T)$, which is associated with the situation in which the profile cut from the Gaussian intensity distribution can be approximated by a top hat distribution with a constant differential reduced brightness of $B_{\text {diff }}(0)$.

\section{QUANTIFICATION OF PROBE CURRENT-PROBE SIZE RELATION}

With the practical brightness of the source known, Eq. (6) gives the desired relation between probe current and source image size. Because the probe in a system is larger than the source image, because of contributions from aberrations and diffraction (electron sources), it is convenient to have the relation between probe current and total probe size, for which aberration and diffraction contributions have to be added to the source image size. Too often, a simple quadrature addition of inconsistently defined diameters is used, with each diameter representing one of the contributions (addition of, e.g., a FWHM for the source image and a FW100 for spherical aberration).

We advocate the use of a consistently defined diameter for all contributions and discourage the use of the quadrature addition. A common mistake for electron probes is the quadrature addition of (consistently defined) FWHMs of the source image and the diffraction limited spot, with the argument that both are (approximately) Gaussian. This would be valid if the source image and the diffraction limited spot are independent distributions that can simply be multiplied to yield the total probe distribution, but this is not so: the total probe is a convolution of the source image and the point source image that contains the blur due to diffraction and aberrations. Using only FW50 diameters, the so-called RPS method fits the full simulations ${ }^{34}$ better than the quadrature addition. Following the RPS method, the minimum FW50 total probe size at the target is

$$
d_{p}=\sqrt{\left(\left(d_{A}^{4}+d_{S}^{4}\right)^{1.3 / 4}+d_{\mathrm{I}}^{1.3}\right)^{2 / 1.3}+d_{C}^{2}} .
$$

Each of the individual terms is the exact FW50 value that would exist in the absence of the other three. The powers are simply those that give a good approximation to complete simulations of electron or ion probes. The FW50 source image diameter $d_{\mathrm{I}}$ follows from Eq. (6), and the other diameters represent the diffraction contribution, $d_{A}$, and the contribu-

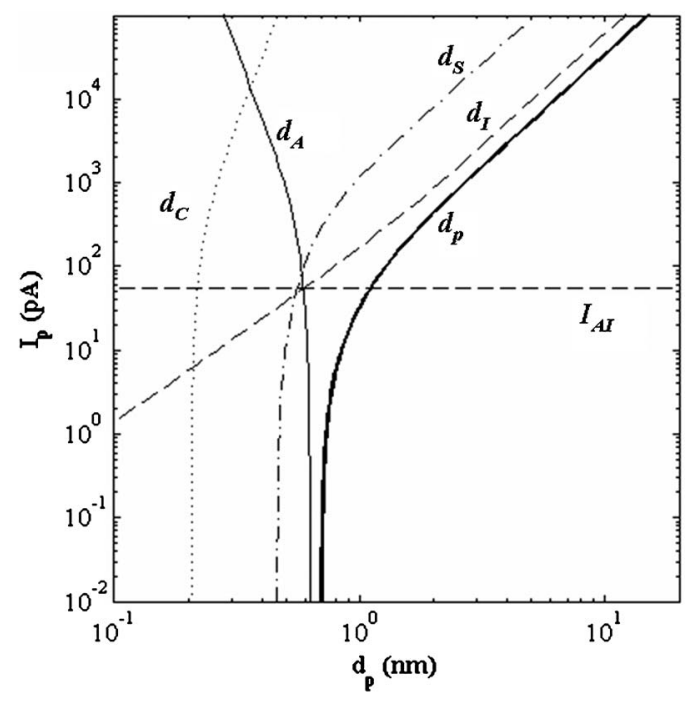

FIG. 5. Typical probe size-probe current relation calculated with Eqs. (6)-(10). $B=5 \times 10^{7} \mathrm{~A} / \mathrm{m}^{2} \mathrm{sr} \mathrm{V}, \Delta E_{\mathrm{FW} 50}=0.6 \mathrm{eV}, V_{p}=30 \mathrm{kV}, C_{s}=12 \mathrm{~mm}$, and $C_{c}=4.4 \mathrm{~mm}$.

tions from chromatic and spherical aberration, $d_{C}$ and $d_{S}$, respectively,

$$
\begin{aligned}
& d_{A}=\frac{0.66 \times 10^{-9}}{\sqrt{V_{p}} \alpha_{p}}, \\
& d_{C}=0.6 C_{C} \frac{\Delta E_{\mathrm{FW} 50}}{V_{p}} \alpha_{p}, \\
& d_{S}=0.18 C_{S} \alpha_{p}^{3} .
\end{aligned}
$$

$C_{C}$ and $C_{S}$ are the chromatic and spherical aberration coefficients of the system. When the last probe forming lens highly demagnifies the aberrations of preceding lenses, as is usually the case, it suffices to use the coefficients of the last lens. $\Delta E_{\mathrm{FW} 50}$ in Eq. (20) is the FW50 of the energy distribution of the electrons. It is noted that $\Delta E_{\mathrm{FW} 50}$ (affecting $d_{C}$ ) and $B$ (affecting $d_{\mathrm{I}}$ ) depend on the same emission parameters, and cannot be tuned independently.

Because of the opposite dependence of $d_{\mathrm{I}}$ and $d_{A}$ vs $d_{C}$ and $d_{S}$ on $\alpha_{p}$, there is, for each desired combination of probe current and beam energy, a different optimum angle that minimizes the total probe size. Figure 5 shows an example of the optimum probe current-probe size relation for an imaginary SEM and a beam energy of $30 \mathrm{kV}$.

For each point on the $d_{p}$ curve in Fig. 5, the beam halfopening angle at the target was optimized (here, from $\sim 6$ to $\sim 14 \mathrm{mrad}$ ). Probe currents much less than $1 \mathrm{pA}$ are not practical, but we include these to illustrate how fast the initial rise of $I_{p}$ with $d_{p}$ is. We did not include probe currents $>20 \mathrm{nA}$ because in this example, we have neglected the gun aberrations which become important at larger probe currents, when the acceptance angle at the gun has to become large. ${ }^{35}$

As can be seen, the probe current for which the contributions from diffraction and source image have the same size 
$\left(d_{A}=d_{\mathrm{I}}\right)$ is a useful characteristic. Because $d_{\mathrm{I}}$ and $d_{A}$ have the same beam energy and angle dependence this probe current $I_{A \mathrm{I}}$ is determined solely by the practical brightness of the source: $I_{A \mathrm{I}}=1.08 \times 10^{-18} \mathrm{~B}$. This brightness current $I_{A \mathrm{I}}$ separates the different regimes that can be distinguished in Fig. 5: for $I_{p}<I_{A \mathrm{I}}$, the contributions $d_{A}$ and $d_{\mathrm{I}}$ balance $d_{S}$, while for $I_{p}>I_{A \mathrm{I}}, d_{A}$ becomes negligible, and the balance is between $d_{\mathrm{I}}$ and $d_{S}$. Note that, at lower beam energy, $d_{C}$ will play a more important role.

In general, each section of the $I_{p}\left(d_{p}\right)$ curve has its own figure of merit for source and/or column properties, which can be found from the equations. Increasing the right figure of merit will improve the system performance for the curve section of interest. In summary, the practical brightness of the source in combination with the RPS method allows us to quantify the relation between probe current and probe size for a probe forming system and find the performancelimiting factors for each application.

\section{CONCLUSION}

In probe forming systems such as scanning (transmission) electron microscopes and focused ion beam microscopes, the whole of the virtual source is imaged onto the target. By introducing the practical brightness, the amount of current in the FW50 diameter of that source image can now finally be quantified.

The practical brightness of a source is given by the angular intensity of the source measured at the extractor divided by the product of the area that contains $50 \%$ of the current in the virtual source and the extraction voltage. The practical brightness of a source is determined by the properties of the emitting surface and possible Coulomb interactions between emitter and extractor.

When Coulomb interactions between emitter and extractor are negligible, thermionic, Schottky, and cold field emitters have a virtual source with a Gaussian intensity profile. This yields for the practical brightness in terms of the current density at the emitting surface: $1.44 e j /(\pi k T)$ for thermionic and Schottky sources and $1.44 e j /(\pi d)$ for cold field emitters. This is $1.44 \times$ larger than the axial differential reduced brightness for these source types. For emitters with unknown source intensity profile, the practical brightness can only be measured.

Without (additional) electron-electron interactions in the column, or changes of the source intensity profile, the practical brightness is conserved from the extractor down to target, unaffected by the quality of the column: the probe current in the source image is proportional to the practical brightness of the source. The total probe size will be an enlargement of the source image, which does depend on the quality of the particular column, and on the energy spread of the electrons in the beam. For electron beams, diffraction gives an additional contribution.

Because the practical brightness is the only brightness that can actually quantify the amount of current in a probe, we hope that, for future characterizations of probe forming systems, the practical brightness will replace the use of all other brightness definitions.

${ }^{1}$ L. W. Swanson and G. A. Schwind, in Handbook of Charged Particle Optics, edited by J. Orloff (CRC, New York, 1997), Chap. 2, p. 77.

${ }^{2}$ P. W. Hawkes and E. Kasper, Principles of Electron Optics (Academic, London, 1996), Vol. 2, Chap. 47, p. 971

${ }^{3}$ I. Müllerová, Scanning Microsc. 13, 7 (1999).

${ }^{4}$ D. C. Joy, in Characterization and Metrology for ULSI Technology 2005, Richardson, TX, 15-18 March 2005, edited by D. G. Seiler (American Institute of Physics, Melville, 2005) Paper No. CP 788, p. 535.

${ }^{5}$ Y. Neo, H. Mimura and T. Matsumoto, Appl. Phys. Lett. 88, 073511 (2006).

${ }^{6}$ J. H. Fink and B. W. Schumacher, Nucl. Instrum. Methods 130, 353 (1975).

${ }^{7}$ A. C. Crewe, in Handbook of Charged Particle Optics, edited by J. Orloff (CRC, New York, 1997), Chap. 10, p. 406

${ }^{8}$ L. Reimer, Scanning Electron Microscopy: Physics of Image Formation and Microanalysis (Springer, Berlin, 1998), Chap. 2, p. 53.

${ }^{9}$ G. H. Jansen, Advances in Electronics and Electron Physics (Academic, Boston, 1990), Vol. 21.

${ }^{10}$ J. E. Barth, M. D. Nykerk, Nucl. Instrum. Methods Phys. Res. A 427, 86 (1999).

${ }^{11}$ T. Radlička and B. Lencová, Ultramicroscopy 108, 445 (2008).

${ }^{12}$ A. H. V. van Veen, C. W. Hagen, J. E. Barth, and P. Kruit, J. Vac. Sci. Technol. B 19, 2038 (2001).

${ }^{13}$ N. de Jonge, J. Appl. Phys. 95, 673 (2004)

${ }^{14}$ A. E. Vladår, Z. Radi, M. T. Postek, and D. C. Joy, Scanning 28, 133 (2006).

${ }^{15}$ X. R. Jiang, Thesis Delft University, 1996.

${ }^{16} \mathrm{P}$. W. Hawkes and E. Kasper, Principles of Electron Optics (Academic, London, 1996), Vol. 2, Chap. 44, p. 924.

${ }^{17}$ J. W. Ward, R. L. Kubena, and M. W. Utlaut, J. Vac. Sci. Technol. B 6, 2090 (1988).

${ }^{18}$ S. A. Rishton, S. P. Beaumont, and C. D. W. Wilkinson, J. Phys. E 17, 296 (1984).

${ }^{19}$ E. Kratschmer, S. A. Rishton, D. P. Kern, and T. H. P. Chang, J. Vac. Sci. Technol. B 6, 2074 (1988).

${ }^{20}$ S. Babin, M. Gaevsky, D. Joy, M. Machin, and A. Martynov, J. Vac. Sci. Technol. B 24, 2956 (2006).

${ }^{21}$ M. J. Fransen, E. P. N. Damen, C. Schiller, T. L. van Rooy, H. B. Groen, and P. Kruit, Appl. Surf. Sci. 94-95, 107 (1996).

${ }^{22}$ D. C. Joy, J. Microsc. 208, 24 (2002).

${ }^{23}$ A. S. Carasso, D. S. Bright, and A. E. Vladár, Opt. Eng. (Bellingham) 41, 2499 (2002).

${ }^{24}$ J. A. Liddle, P. Naulleau, and G. Schmid, J. Vac. Sci. Technol. B 22, 2897 (2004).

${ }^{25}$ M. J. Fransen, M. H. F. Overwijk, and P. Kruit, Appl. Surf. Sci. 146, 357 (1999).

${ }^{26}$ D. Tuggle, N. K. Kang, and L. W. Swanson, Optik (Jena) 63, 313 (1983).

${ }^{27}$ R. R. Law, Proc. IRE 25, 954 (1937).

${ }^{28}$ J. Worster, Br. J. Appl. Phys. 2, 889 (1969).

${ }^{29}$ J. C. Wiesner and T. E. Everhart, J. Appl. Phys. 44, 2140 (1973).

${ }^{30}$ S. Fujita and H. Shimoyama, J. Electron Microsc. 54, 331 (2005).

${ }^{31}$ S. Fujita and H. Shimoyama, J. Electron Microsc. 54, 413 (2005).

${ }^{32}$ S. Fujita and H. Shimoyama, J. Vac. Sci. Technol. B 24, 1891 (2006).

${ }^{33}$ A. B. El-Kareh, J. C. Wolfe, and J. E. Wolfe, J. Appl. Phys. 48, 4749 (1977).

${ }^{34}$ J. E. Barth and P. Kruit, Optik (Jena) 101, 101 (1996).

${ }^{35}$ P. Kruit, M. Bezuijen, and J. E. Barth, J. Appl. Phys. 99, 024315 (2006). 